\title{
Application of the Multiscale Finite Element Method to the numerical modeling of regional land subsidence
}

\author{
Shujun Yea , Yuqun Xue ${ }^{a}$, Jichun Wu ${ }^{a}$, Hanmei Wang ${ }^{b}$, Zixin Wei ${ }^{b}$ and Xuexin Yan \\ ${ }^{a}$ Key Laboratory of Surficial Geochemistry, Ministry of Education; School of Earth Sciences and \\ Engineering, Nanjing University, Nanjing, P.R. China \\ Email: sjye@nju.edu.cn \\ ${ }^{b}$ Shanghai Institute of Geology Survey, Shanghai, China
}

\begin{abstract}
For a regional land subsidence problem, there are difficulties for the traditional finite element method to solve the regional groundwater flow equation involved. A new finite element method - multiscale finite element method (MsFEM) applied to resolve three dimensional groundwater flow problem- is introduced. Three advantages make the MsFEM very useful for groundwater flow simulation in a large region with highly heterogeneous porous media. One advantage is that this method can significantly save computing efforts by coarse grid mesh. The variation of parameters in an element is brought to the multiscale base functions. Second one is that this method can to a large extent avoid large aspect ratios of elements by combining aquifers and aquitards in one element. The third advantage is that the hydraulic heads in different depths of aquitards or aquifers could be rather accurately interpolated using the multiscale base functions and the nodal heads of the element by MsFEM. The third advantage is useful for the subsidence modeling. A numerical example is done to verify the advantages of MsFEM. Finally, the MsFEM is applied to solve the regional land subsidence model of Shanghai, which includes a three-dimensional groundwater flow mathematical model and a one-dimensional subsidence model. The traditional two-step approach is followed in this study, with the hydrodynamics of the pumped multiaquifer system first simulated by a 3-D groundwater flow model and the subsidence then computed with a 1-D subsidence model with the pore pressure field specified as an external distributed source of strength within the porous medium. The simulation period is from March, 1986 to December, 1998. Using MsFEM, the aquifer system in Shanghai is divided into six layers, which means one aquifer and one aquitard are in one layer with an average thickness of about 50 meters. Every element in MsFEM is subdivided into 7 sub-layers in the vertical direction and every horizontal layer in an element is divided into 4 hexahedral elements. So each element is subdivided into 28 hexahedral sub-cells, which allows the heterogeneity of aquifers and aquitards to be considered by multiscale base functions. The aquifers system in total is discretized into 19,996 elements with a total of 25,000 nodes. The hydraulic heads in any depth of aquitards or aquifers could be interpolated using the multiscale base functions and the nodal heads of the element. It is very useful for the settlement calculation for the aquitards or aquifers after hydraulic heads at nodes of elements are obtained by MsFEM. The application of MsFEM significantly saves computing efforts, effectively decreases the aspect ratios of elements and obtains good results, which proves the MsFEM can be applied to solve the real regional 3D groundwater flow problems, and it is an effective numerical method for regional land subsidence modeling.
\end{abstract}

Keywords: $\quad$ Multiscale finite element method, Numerical modeling, Regional Land subsidence 


\section{INTRODUCTION}

Land subsidence caused by excessive groundwater withdrawal has occurred in many regions in the world. In China, land subsidence is becoming a serious environmental problem, occurring mainly in 17 provinces (cities) located in the eastern and central regions, with a total subsidence area of more than $9 \times 10^{4} \mathrm{~km}^{2}$ (Xue et al. 2005). The Yangtse Delta and the Huang-huai-hai Plain are the two biggest and most serious land subsidence areas in China. By the end of the last century, the area where the cumulative subsidence exceeded $200 \mathrm{~mm}$ in the Yangtse Delta was approximately $10,000 \mathrm{~km}^{2}$. Land subsidence has occurred over almost all of $50,000 \mathrm{~km}^{2}$ of the Huang-huai-hai Plain. Such large land subsidence regions have never been reported out of China.

Land subsidence model includes ground water flow model and subsidence model. In this paper the traditional two-step approach is followed, with the hydrodynamics of the pumped multiaquifer system first simulated by a 3-D groundwater flow model and the subsidence then computed with the aid of a 1-D subsidence model with the pore pressure field specified as an external distributed source of strength within the porous medium.

Regional groundwater flow modeling is the basis for simulating the regional land subsidence, because the pore pressure change from the groundwater flow model is used as input data to the subsidence model. Several issues have made the large regional groundwater modeling quite a difficult task. One issue is the study areas are very large, many aquifers and aquitards are to be modeled, and the aquifers and aquitards are highly heterogeneous. Three dimensional (3D) groundwater flow mathematical model is usually developed for the simulation of regional land subsidence because of the anisotropic and highly heterogeneous porous media, different compressibility of aquifers and aquitards and also the 3D groundwater flow. It is difficult to apply the FDM or traditional FEM to solve the 3D groundwater flow mathematical model for land subsidence regions like the Yangtse Delta and the Huang-Huai-Hai Plain. Because very many computer resources and long computational time are required. Second issue is the much greater horizontal extent than the vertical extent of the large regional ground water flow modeling.

Another important problem of the large regional land subsidence modeling is the suitable way to accurate calculate the hydraulic heads in aquitards. Mostly the land subsidence is caused by the settlement of aquitards which are usually composed by clay media. The aquitards can be very heterogeneous along the vertical direction and have to be divided into several soil layers according to its lithology. For example, the first aquitard in Yangtse Delta area was the main compressible layer there in the history. It is 6-15m thick, composed of two different soft soil layers, and a hard soil layer. The aquitard have to be divided into several different layers because of the heterogeneity when the traditional FEM is applied, which will further worsen the two problems of a lot of computation efforts and elements with large aspect ratios.

As mentioned above, it is difficult to apply the traditional finite element method to solve the regional three dimensional groundwater flow model when doing the regional land subsidence simulation. Therefore it's necessary to find a new numerical method that can decrease the number of elements, overcome the problem of large aspect ratios of elements, and give accurate results. The multiscale finite element method (MsFEM) (Ye et al. 2004) applied in this paper can serve these purposes.

\section{ADVANTAGES OF THE MSFEM IN SOLVING GROUNDWATER FLOW MODELS}

The MsFEM has the advantages of decreasing the number of elements by including heterogeneous media in one element to save the computing efforts and overcoming high aspect ratios of elements to gain accurate results. Another advantage of MsFEM is that the hydraulic heads in different depths of aquitards and aquifers could be rather accurately interpolated using multiscale base functions and nodal heads of the element. Details of MsFEM can refer to the paper by Ye et al. (2004). One numerical example is done below to verify these advantages.

The study area is a rectangular domain covering $10 \mathrm{~km} \times 10 \mathrm{~km} \times 280 \mathrm{~m}$ (Figure 1a). The simulated aquifer system is composed of seven confined aquifers and seven aquitards (Figure 1b). The aquifers and aquitards are distributed in turns in the vertical direction. All the aquifers and aquitards are 20 meters thick and are all heterogeneous. Hydraulic conductivities from left to right in all aquifers vary gradually from $1 \mathrm{~m} / \mathrm{d}$ to $196 \mathrm{~m} / \mathrm{d}$, which means they increase $5 \mathrm{~m} / \mathrm{d}$ every 250 meters from left to right. Hydraulic conductivities from left to right in all aquitards vary gradually from $0.005 \mathrm{~m} / \mathrm{d}$ to $0.98 \mathrm{~m} / \mathrm{d}$, which means they increase $0.025 \mathrm{~m} / \mathrm{d}$ every 250 meters from left to right. The left and right sides of the area are modeled as first type boundaries with the head on the left side is $10 \mathrm{~m}$, and head on the right side is $1 \mathrm{~m}$. Other sides are modeled as impermeable boundaries. MsFEM and traditional finite element method (FEM) are used to solve this 
example problem. The mathematical model has no analytical solution, so the fine scale results obtained by the traditional finite element method with fine elements (FEM-F) are regarded as the exact solutions. Using FEM-F, the study area is divided into 14 layers in the vertical direction, which means the aquifers are separated from the aquitards. Every horizontal layer is divided into 1,600 hexahedral elements. Therefore the study area is divided into 22,400 hexahedral elements and 25,215 nodes. Using MsFEM, the study area is divided into 2 layers in the vertical direction, which means 4 aquifers and 3 aquitards in the first layer and 4 aquitards and 3 aquifers in the second layer. Every horizontal layer is divided into 400 hexahedral elements. Therefore the study area is divided into 800 hexahedral elements and 1,323 nodes. In order to compute the base function numerically, every element in MsFEM is subdivided into 7 layers in the vertical direction, which means the aquifers are separated from the aquitards, divided into 4 hexahedral elements for each horizontal layer, and then subdivided into 28 hexahedral elements (Figure 1c). Using FEM, the study area is divided into 14 layers in the vertical direction. Every horizontal layer is divided into 400 hexahedral elements. Therefore the study area is divided into 5,600 hexahedral elements and 6,615 nodes. The elements in FEM are homogeneous by averaging the hydraulic conductivities in the elements.

The relative errors of the results of FEM and MsFEM compared to these of FEM-F in the interface $y=5,000 \mathrm{~m}$ and $\mathrm{z}=-140 \mathrm{~m}$ are shown in Figure 2. All the results in Figure 2 are the calculated heads at the nodes of elements. Table 1 shows the comparison of the number of nodes, CPU time ( by the PC of Intel(R) Core TM 2 Quad CPU Q6600@2.40GHz, 2.39GHz, 3.23GB Memory), ratio of horizontal extent to vertical extent of elements, and relative errors of results of MsFEM, FEM and FEM-F. From Figure 2 and table 1, one can see that accurate results are gained by MsFEM with much less computing memory -19 times less number of nodes, much less CPU time - 64 times faster, and 3.5 times less aspect ratios of elements for the problem.

Although 4 aquifers and 3 aquitards are put in one element in the first layer and 4 aquitards and 3 aquifers are put in one element in the second layer by MsFEM, the hydraulic head in any point in the element can be rather accurate interpolated by the multiscale base functions which can capture the small scale heterogeneity

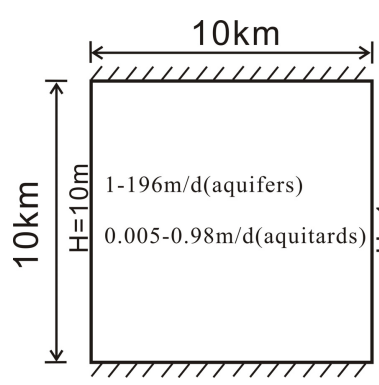

(a)

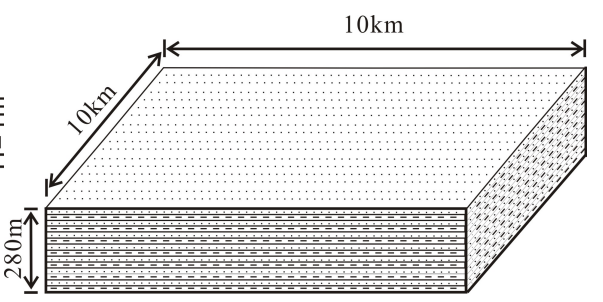

(b)

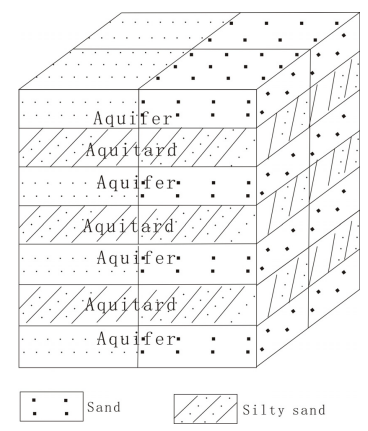

(c)

Figure 1. Illustration of the numerical example

(a) 2-D horizontal diagram (b) 3-D diagram (c) subgrid of an element

information. Figures 3 shows the relative errors between the interpolated heads by MsFEM and the nodal heads by FEM-F on section $y=2,500 \mathrm{~m}$ and $\mathrm{z}=-60 \mathrm{~m}$. Figures 3 also shows the relative errors between the nodal heads by FEM and FEM-F on section $y=2,500 \mathrm{~m}$ and $\mathrm{z}=-60 \mathrm{~m}$. The relative errors of the results of FEM are large as shown in Figures 2. It is caused by the coarse grid which simplifies the heterogeneity information and has large ratio of horizontal extent to vertical extent. We can find that the interpolated heads by MsFEM are very close to the nodal heads by FEM-F, which means the head at any point in the elements can be rather accurately interpolated by the nodal heads and the multiscale base functions.

Table 1. Comparison of different numerical methods

\begin{tabular}{|l|l|l|l|l|}
\hline Method & $\begin{array}{l}\text { Number of } \\
\text { Nodes }\end{array}$ & $\begin{array}{l}\text { CPU time } \\
\text { (second) }\end{array}$ & $\begin{array}{l}\text { Ratio of horizontal extent } \\
\text { to vertical extent of } \\
\text { elements }\end{array}$ & $\begin{array}{l}\text { Maximum } \\
\text { relative error (\%) }\end{array}$ \\
\hline FEM-F & 25,215 & 278.48 & 12.5 & 0 \\
\hline FEM & 6,615 & 4.97 & 25 & 29.68 \\
\hline MsFEM & 1,323 & 4.38 & 3.57 & 3.15 \\
\hline
\end{tabular}


So the hydraulic heads in different depths of aquitards or aquifers could be rather accurately interpolated using the multiscale base functions and the nodal heads of the element by MsFEM without dividing the heterogeneous aquitards or aquifers into several layers of elements. The interpolated heads can be further used to effectively calculate the settlement of aquitards or aquifers by the subsidence model.

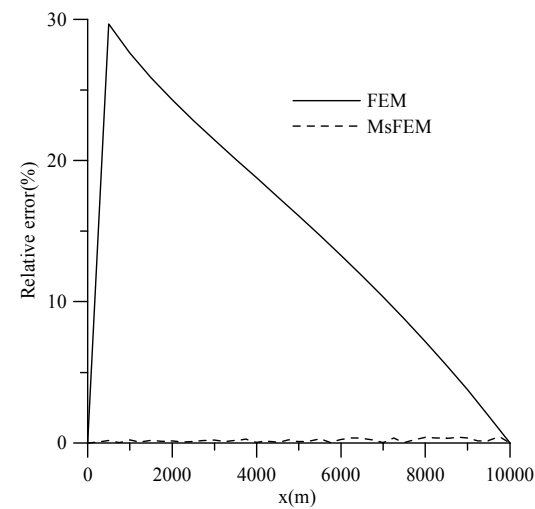

Figure 2. Relative errors of the results of FEM and MsFEM on section $\mathrm{y}=5,000 \mathrm{~m}$ and $\mathrm{z}=-140 \mathrm{~m}$.

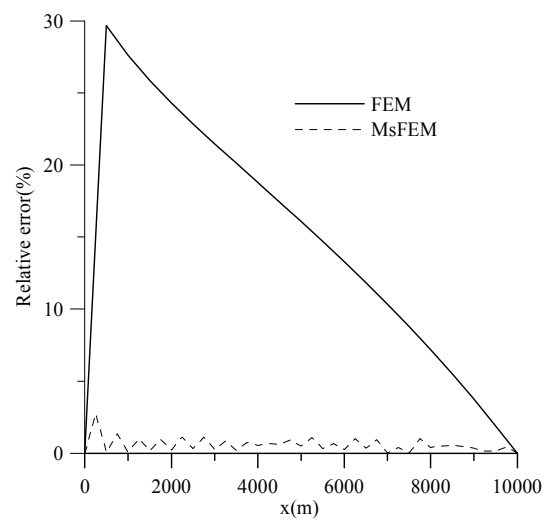

Figure 3. Relative errors of the results of FEM and MsFEM on section $\mathrm{y}=2,500 \mathrm{~m}$ and $\mathrm{z}=-60 \mathrm{~m}$.

\section{APPLICATION OF MSFEM TO LAND SUBSIDENCE MODELING IN SHANGHAI}

The land subsidence in Shanghai induced by excessive groundwater withdrawal was detected in 1921. Shanghai city, lying in the Yangtse Delta, is one of the cities which experienced the most severe land subsidence in China (Figure 4). To simulate the land subsidence in Shanghai, 3-D groundwater flow mathematical model and 1-D subsidence model are developed. The MsFEM is applied to solve the 3-D groundwater flow problem, and then the calculated hydraulic heads is used as input data to 1-D subsidence model.

\subsection{Sedimentary layers in Shanghai}

The study area is Shanghai, with an area of about $5,300 \mathrm{~km}^{2}$. The aquifers system is composed of Quaternary unconsolidated sediments. The sediments are about $200-350 \mathrm{~m}$ thick just lacking in some local places. The bedrock is located under the sediments. The unconsolidated sediments are composed of clay, sandy clay and sand, in layers, in vertical direction. They are divided into aquifers and aquitards according to lithology. The sediments are divided into the phreatic aquifer (A0), first aquitard which can be divided into first soft layer, second soft layer, second hard layer and semi-confined aquifer in some places (B1), first confined aquifer (A1), second aquitard (B2), second confined aquifer (A2), third aquitard (B3), third confined aquifer (A3), fourth aquitard (B4), fourth confined aquifer (A4), fifth aquitard (B5), fifth confined aquifer (A5), and sixth aquitard (B6) (Figure 5). The aquifers and aquitards are not continuous in the study area, and do not exist in some places.

\subsection{Mathematical model of land subsidence}

The traditional two-step approach (Gambolati and Freeze, 1973; Teatini et al., 2011) is followed in the present analysis, with the hydrodynamics of the pumped multiaquifer system first simulated by a 3-D groundwater flow model and the subsidence of Shanghai then computed with a 1-D subsidence model with the pore pressure field specified as an external distributed source of strength within the porous medium. 
The groundwater flow field presents obvious three-dimensional flow in Shanghai. An anisotropic, heterogeneous and three-dimensional groundwater flow mathematical model is developed as follows:

$$
\begin{gathered}
\frac{\partial}{\partial x_{i}}\left(K_{i j} \frac{\partial H}{\partial x_{j}}\right)+\omega=S_{s} \frac{\partial H}{\partial t} \quad i, j=1,2,3 \quad(x, y, z) \in \Omega \\
H(x, y, z, 0)=H_{0} \quad(x, y, z) \in \Omega \\
H(x, y, z, t)=\left.H_{0}\right|_{\Gamma_{1}}=H_{1}(x, y, z, t) \quad t \geq 0,(x, y, z) \in \Gamma_{1} \\
\left.K_{i, j} \frac{\partial H}{\partial n} n_{i}\right|_{\Gamma_{2}}=q(x, y, z, t) \quad t \geq 0,(x, y, z) \in \Gamma_{2}
\end{gathered}
$$

where $H$ is hydraulic head, $K$ is hydraulic conductivity and $S_{s}$ is specific storage, $\omega$ is the source or sink term, $\mathrm{H}_{0}$ is hydraulic head at initial time, $\mathrm{H}_{1}$ is hydraulic head on the first type boundaries, $\Gamma_{1}$ is the first type boundaries, $\mathrm{q}$ is the flow on the second type boundaries, $\Gamma_{2}$ is the second type boundaries, $\mathrm{n}$ is the normal vector of the second type boundary.

There is no obvious horizontal displacement of sediments in Shanghai, so one dimensional vertical displacement of sediments is assumed. The traditional 1-D nonlinear subsidence model is followed in the present analysis.

The time dependent deformation of any aquifer or aquitard is given by

$$
\Delta L=\sum_{j=1}^{m} \gamma \alpha_{j} \Delta H_{j}(t) \Delta Z_{j}
$$

where $\Delta L$ is deformation, $\mathrm{m}$ is the total number of sedimentary layers in an aquitard or aquifer, $\Delta Z_{j}$ is the thickness of the jth sedimentary layer, $\gamma$ is specific weight of water, $\Delta H_{j}$ is the change of hydraulic head in a time period, and $\alpha_{j}$ is volume compressibility of sediments which depends on the hydraulic head as follows

$$
\begin{array}{ll}
\alpha=\alpha_{k e} & \left(H \geq H_{p}\right) \\
\alpha=\alpha_{k v} & \left(H<H_{p}\right)
\end{array}
$$

Where $\alpha_{k e}$ is elastic volume compressibility, $\alpha_{k v}$ is plastic volume compressibility, and $\mathrm{H}_{\mathrm{p}}$ is the hydraulic head corresponding to the preconsolidation stress. The $\alpha$ is related to $\mathrm{S}_{\mathrm{s}}$ by

$$
S_{s}=\gamma(\alpha+n \beta)
$$

Where $\gamma$ is specific weight of water, $n$ is porosity, $\beta$ is volume compressibility of water. For this 1-D subsidence model, it is very simple without using MsFEM. The deformation of any aquifer or aquitard is calculated according to equation (5) with the changes in hydraulic heads calculated by the 3-D groundwater flow mathematical model.

\subsection{Numerical Modeling}

There are 6 aquifers and 6 aquitards which have different thicknesses, with the thin layers being less than 10 $\mathrm{m}$ thick (Figure 5). The horizontal extent of the study area is much larger than the vertical extent.

Using MsFEM, the aquifer system in Shanghai is divided into six layers, which means one aquifer and one aquitard are in one layer with an average thickness of about 50 meters. The aquifer system is discretized into hexahedral elements. The hexahedral elements are mostly square in the horizontal direction, but become irregular quadrangle in some local places like at the observation wells and pumping wells. The size of the element in the horizontal direction is about $1,200 \mathrm{~m} \times 1,200 \mathrm{~m}$. The ratio of horizontal length to the vertical length of the hexahedral element is about 24 . This ratio is a little large, however the aquifers system is not divided into finer elements due to the increase in calculation efforts. The ratio can reach several hundred for some aquifers or aquitards with thicknesses of less than $10 \mathrm{~m}$ if the traditional FEM is applied. Every element in MsFEM is subdivided into 7 sub-layers in the vertical direction and every horizontal layer in an element is 
divided into 4 hexahedral elements. So each element is subdivided into 28 hexahedral subcells, which allows the heterogeneity of aquifers and aquitards to be considered by multiscale base functions. This is the pivotal difference between the MsFEM and the traditional FEM. Subcell grid of each element looks like the one shown in Figure 1c. The spatial distribution of aquifers and aquitards in each element could be different, which depends on the distribution of sediments. For example, the fifth layer of elements include the fourth confined aquifer and the fifth aquitard, and the fourth confined aquifer accounts for 3 to 6 sub-layers and the

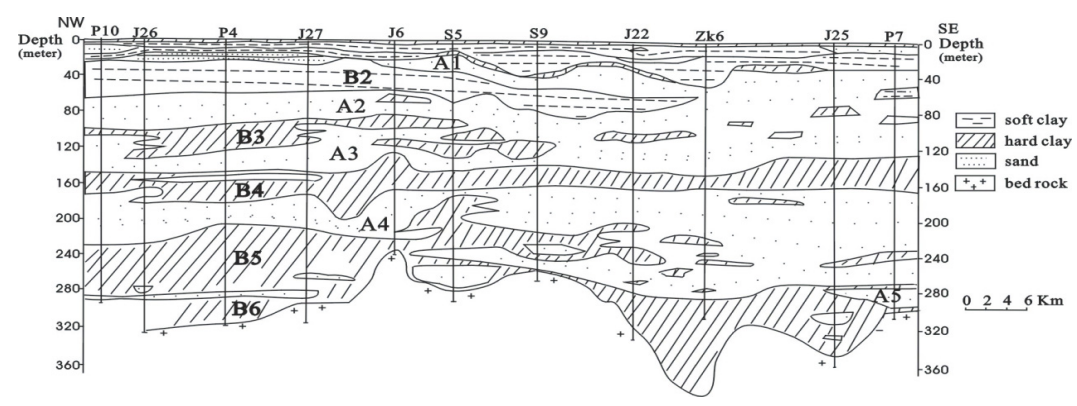

Figure 5. Schematic stratigraphic section in Shanghai. fifth aquitard accounts for 1 to 4 sub-layers in an element.

Each aquifer or aquitard has a different area, so the number of elements in each layer is different as shown in table 2. The aquifers system in total is discretized into 19,996 elements with a total of 25,000 nodes. The hydraulic heads in any depth of aquitards or aquifers could be interpolated using the multiscale base functions and the nodal heads of the element. It is very useful for the aquitards or aquifers to calculate the settlement after hydraulic heads at nodes of elements are obtained by MsFEM.

The simulation period is from March, 1986 to December, 1998. The period is separated into 51 time steps, with each time step being 90 days. The model calibration stage includes the first 35 time steps while the model verification stage includes time step 37 to time step 51. Time unit is day.
Table 2. 3D grid of the aquifers system of Shanghai

\begin{tabular}{|c|c|c|}
\hline No. of layer & Composition & Number of elements \\
\hline 1 & A0 and B1 & 3,476 \\
\hline 2 & A1 and B2 & 3,659 \\
\hline 3 & A2 and B3 & 3,651 \\
\hline 4 & A3 and B4 & 3,639 \\
\hline 5 & A4 and B5 & 3,509 \\
\hline 6 & A5 and B6 & 1,864 \\
\hline
\end{tabular}

The initial time of the flow mathematical model is March 30, 1986. The groundwater levels at the nodes on the top of each element layer are obtained by Kriging interpolation of the groundwater level data of the corresponding aquifers at the initial time, as those on the top of the fifth layer of elements shown in Fig. 9. The initial groundwater levels in the fifth confined aquifer are also assigned to the nodes on the bottom of the sixth layer of elements. There is no observation groundwater level data in the phreatic aquifer prior to 1993. The groundwater levels in the phreatic aquifer on March 30, 1993 are approximately considered as the initial water levels, because the groundwater level didn't change drastically over many years without withdrawal. The subsidence model has the same initial time as the flow model and the vertical displacements of all the nodes are zero initially in the subsidence model.

The first type boundary conditions of each layer for the flow mathematical model are obtained by Kriging interpolation of the observation well data close to the boundaries. The top boundary of the flow mathematical model is the phreatic aquifer and the bottom boundary is bedrock which is assumed as a no flow boundary. The top of the vertical one dimensional subsidence model is land surface which is a free surface, and the bottom of the subsidence model is bedrock which is a confined surface without displacement.

The model is calibrated and verified against historical records of hydraulic heads and land subsidence. This is done by trial and error with the hydromechanical parameters of the groundwater system accommodated as follows. First the groundwater flow model is validated so as to reproduce the head behavior in time. Then the head change from the 3-D flow model is used as the input data to the 1-D subsidence model. The simulated land subsidence and sediment settlement are verified against bench mark and extensometer records, with the procedure iteratively repeated until a satisfactory match is achieved.

The results from the subsidence model are compared with the available bench mark measurements in Figure 6 showing the computed land subsidence and the measured settlement in Shanghai over the period 1986- 
1998. The agreement of land subsidence distribution is deemed to be satisfactory if considering some land subsidence caused by high building construction or some underground engineering activities.
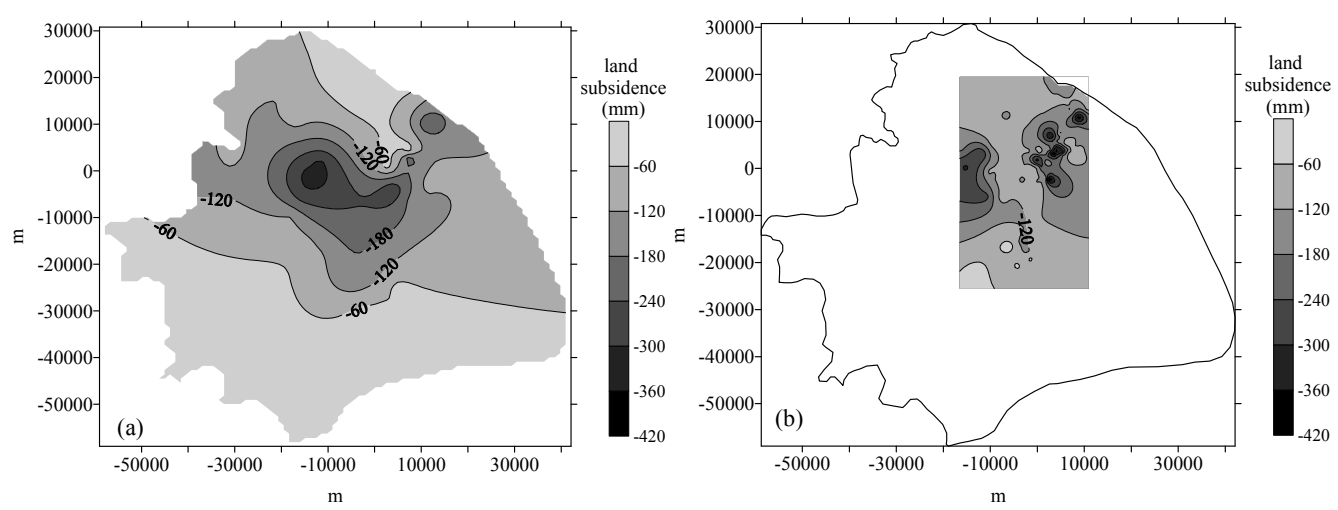

Figure 6. Simulated land subsidence (a) and measure land subsidence (b) over the simulation time.

\section{CONCLUSIONS}

To simulate the regional land subsidence problem, the MsFEM is introduced to solve the regional threedimensional groundwater flow mathematical model of the regional land subsidence mathematical model in this paper. The MsFEM has the advantages of decreasing the number of elements by including heterogeneous media in one element to save the computing efforts and overcoming the high aspect ratios of elements to gain accurate results. Another advantage of MsFEM is that the hydraulic heads in different depths of aquitards and aquifers could be rather accurately interpolated using multiscale base functions and nodal heads of the element. It is important to fatherly obtain good results of the settlement of aquitards and aquifers with obvious heterogeneity along the vertical direction, and at the same time avoid the problems of significant computational time and memory and high aspect ratios of elements of the traditional FEM. These advantages are verified by a numerical example. The MsFEM is applied to solve the 3D groundwater flow mathematical model of Shanghai which is the important part of land subsidence mathematical model. It significantly saves computing efforts, effectively decreases the aspect ratios of elements and obtains good results, which proves the MsFEM can be applied to solve the real regional 3D groundwater flow problems, and it is an effective numerical method for regional land subsidence modeling.

\section{ACKNOWLEDGMENTS}

The paper is financially supported by NSFC No. 41272259.

\section{REFERENCES}

Gambolati, G. and Freeze, R.A. (1973). Mathematical simulation of the subsidence of Venice:1. Theory. Water Resources Research, 9(3): 721-733.

Teatini, P., Castelletto, N., Ferronato, M., Gambolati, G., and Tosi, L. (2011), A new hydrogeologic model to predict anthropogenic uplift of Venice. Water Resources Research, 47, W12507, doi:10.1029/2011WR010900.

Xue, Y.Q., Zhang, Y., Ye, S.J. et al (2005). Land subsidence in China. Environmental Geology, 48(6): 713720.

Ye, S.J., Xue, Y. and Xie, C. (2004). Application of the multiscale finite element method to flow in heterogeneous porous Media. Water Resources Research ,40, W09202, doi:10.1029/2003WR002914. 